\title{
EXPERIENCES OF UAV SURVEYS APPLIED TO ENVIRONMENTAL RISK MANAGEMENT
}

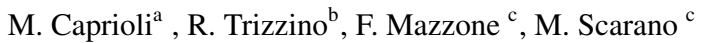 \\ a DICATECh, Politecnico di Bari, Via E. Orabona, 4, 70125 Bari, mauro.caprioli@ poliba.it \\ ${ }^{\mathrm{b}}$ CNR IRPI, Via Amendola, 126/i, 70126 Bari, r.trizzino@ba.irpi.cnr.it \\ ${ }^{\mathrm{c}}$ University of Naples Parthenope, Centro Direzionale, Isola C4, 80133 Napoli, name.surname@ uniparthenope.it
}

Commission I, WG V/b

KEY WORDS: UAV, Photogrammetry, Laser, Risk analysis

\begin{abstract}
:
In this paper the results of some surveys carried out in an area of Apulian territory affected by serious environmental hazard are presented. Unmanned Aerial Vehicles (UAV) are emerging as a key engineering tool for future environmental survey tasks. UAVs are increasingly seen as an attractive low-cost alternative or supplement to aerial and terrestrial photogrammetry due to their low cost, flexibility, availability and readiness for duty. In addition, UAVs can be operated in hazardous or temporarily inaccessible locations, that makes them very suitable for the assessment and management of environmental risk conditions. In order to verify the reliability of these technologies an UAV survey and A LIDAR survey have been carried outalong about $1 \mathrm{~km}$ of coast in the Salento peninsula, near the towns of San Foca, Torre dellOrso and SantAndrea( Lecce, Southern Italy). This area is affected by serious environmental risks due to the presence of dangerous rocky cliffs named falesie. The UAV platform was equipped with a photogrammetric measurement system that allowed us to obtain a mobile mapping of the fractured fronts of dangerous rocky cliffs. UAV-images data have been processed using dedicated software (AgisoftPhotoscan). The point clouds obtained from both the UAV and LIDAR surveys have been processed using Cloud Compare software, with the aim of testing the UAV results with respect to the LIDAR ones. The total error obtained was of centimeter-order that is a very satisfactory result. The environmental information has been arranged in an ArcGIS platform in order to assess the risk levels. The possibility to repeat the survey at time intervals more or less close together depending on the measured levels of risk and to compare the output allows following the trend of the dangerous phenomena. In conclusion, for inaccessible locations of dangerous rocky bodies the UAV survey coupled with GIS methodology proved to be a key engineering tool for the management of environmental risks.
\end{abstract}

\section{INTRODUCTION}

Unmanned Aerial Vehicle (UAV) systems are increasingly seen as an attractive low-cost alternative or supplement to aerial and terrestrial photogrammetry due to their low cost, flexibility, availability and readiness for duty. In addition, UAVs can be operated in hazardous or temporarily inaccessible locations. The combination of photogrammetric aerial and terrestrial recording methods using a mini UAV (also known as drone) opens a broad range of applications, such as surveillance and monitoring of the environment and infrastructural assets (Zhang, 2009; Caprioli et al., 2015). Our research presents an application of UAV surveys to the stability analysis of a typical costal area of Southern Italy that is subject to widespread instability phenomena due to the presence of steep rocky cliffs. The study area is located in the Salento peninsula, in proximity of the towns of San Foca, Torre dellOrso and Sant Andrea, in the province of Lecce (Apulia region, Southern Italy) (Fig. 1). Here, the rocky cliffs are cut in weathered and fractured carbonate rocks affected by intense erosion and show different types of landslides, with various complex mechanisms of rock slope failure. The morphology of rocky coasts is the result of the interaction of numerous natural and anthropogenic processes. In the study area the environmental hazard derives from the possibility of rock falls. So, to assess the risk level of this kind of coastal area it is necessary to map the structural set up, the discontinuity pattern and density and the degree of weathering of rock masses. The main types of erosional and landslide processes observed along the coastal stretch investigated are related to slides, falls and topples. The waves attack the weak part of the rock and leave the resistant areas to form the different fea-

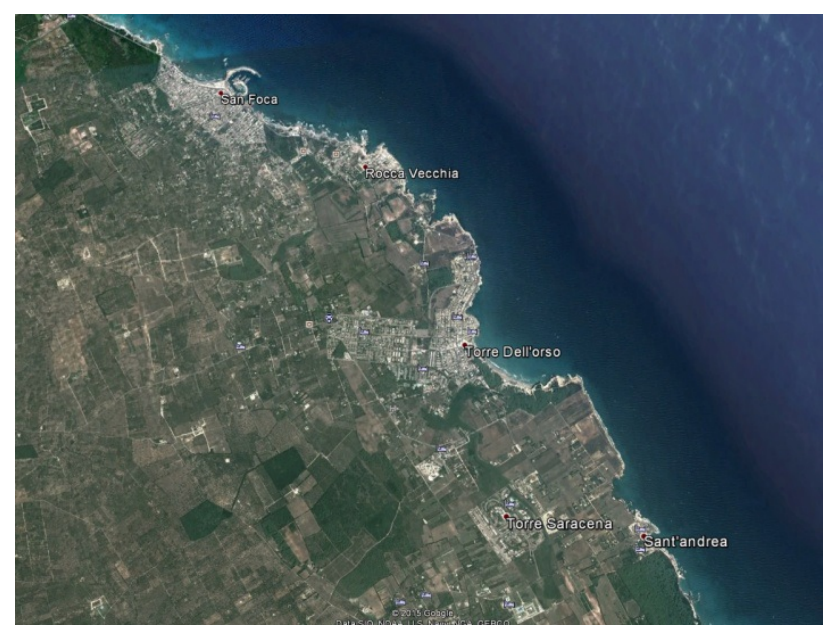

Figure 1: The study area (Google Earth)

tures. Processes of erosion are more active along joint systems. Wave energy causes gouge or hollow forming caves and arches which are continually widened by erosion and weathering. The roof becomes too heavy and collapses forming a stack. The stack is then eroded and may form a stump. Falls of blocks and big slices of rocks are triggered by undercutting at the base of the cliff. Tension cracks and fractures control their detachment. Toppling of blocks mainly occurs along failures parallel to the coastline causing cliff retreating (Fig. 2). In this context the landbased mapping of the dangerous natural structures is very diffi- 


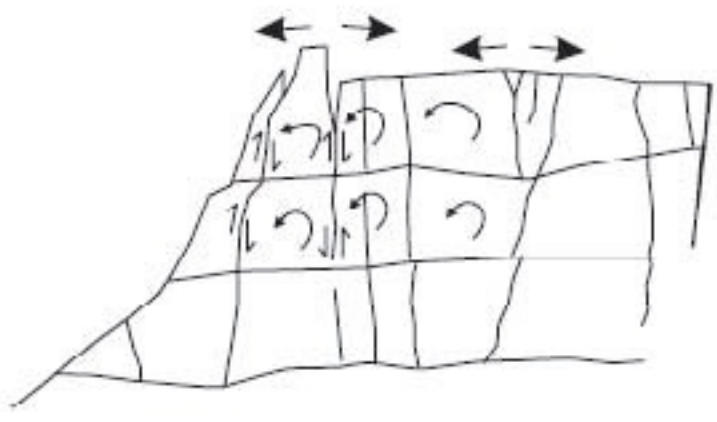

Figure 2: Toppling failure mechanism (after Hoek et al., 1977)

cult and time and resources expending. Today, UAVs can be used as a precise, automated and computer controlled data acquisition and measurement platform in in hazardous or temporarily inaccessible locations, thanks to the recent developments of low-cost sensors such as off-the-shelf digital cameras, GPS/INS (Global Positioning System / Inertial Navigation System) based stabilization, navigation units and laser scanners. The UAV platform was equipped with a shot measurement system that allowed us to obtain a mobile mapping of the fractured fronts of dangerous rocky cliffs.

\section{THE UAV SURVEY}

The UAV survey was executed on 22th January 2015 in collaboration with SAL Engineering (Modena, Italy) which carried out three flights at about $69 \mathrm{~m}$ of altitude with a esacopter equipped with a brushless electric motor and a GNSS satellite positioning system. Nearly 1053 nadiral and inclined images have been taken with CANON EOS 550D cameras with $18 \mathrm{mpx}$ APS-C sensor. Thanks to the GPS-Position-Hold technology a framework of 10 Ground Control Point (GCP) has been set on the study area and, for internal use and further error checking, 3 more points (check points) were collected using dual frequency Trimble GPS receivers (FIg. 4). The location of Ground Control Point (GCP) and surrounding check points are reported in Figure 3. A GIS is very effective at recognizing and analysing spatial relationships between mapped features. In order to model aerial surveyed data combining them with other kinds of data, such as geologic maps, we imported the UAV and geo-structural information in GIS layers, to form maps that can display trends or predict various responses of the dangerous rocky cliffs to climatic changes (Burrough, 1986). A major goal of real-time monitoring was to enable discernment between relatively low risk slope creep from higherrisk accelerating motion, which usually precedes catastrophic detachment.

In the present study, we combined imagery, geological and movement data layers to enhance the geotechnical interpretation of the environmental hazardous situations and to advise clients with some reasonable measure of reliability. Once the field survey were done, the collected data have been processed with computation methods of visible-surface representation (Terzopoulos, 1988; Watson et al., 1985). The image processing has been carried out with Agisoft PhotoScan dedicated software. As a result, starting from 1053 nadiral and inclined images, a cloud of 1226867 tie-points with a $0.0106382 \mathrm{~m} /$ pix ground resolution was calculated. The output data have been digitized and georeferenced, and easily put into a GIS and ortho-rectified.

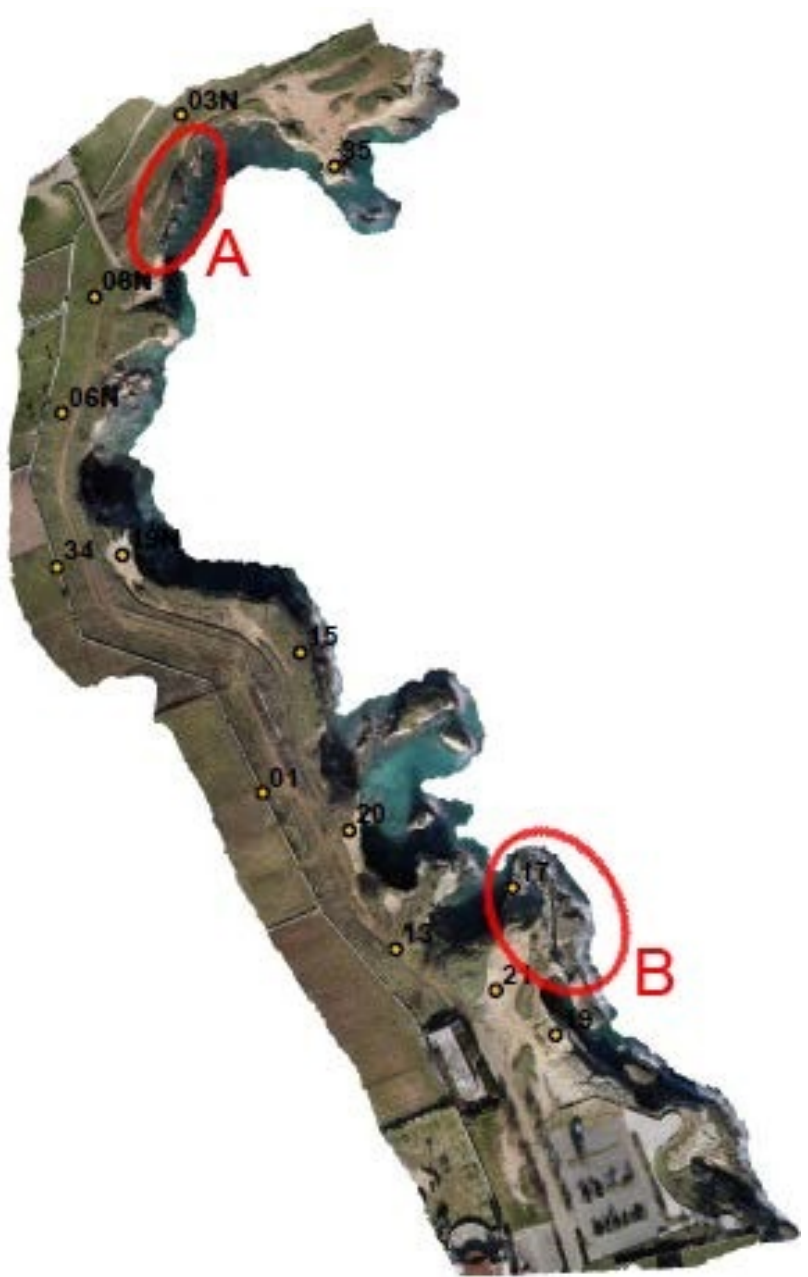

Figure 3: The location of Ground Control Point (GCP)

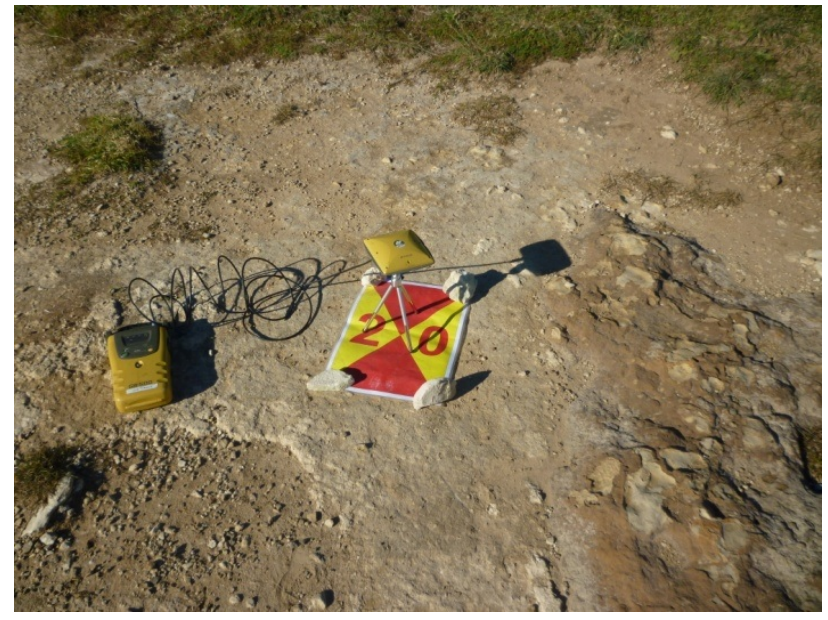

Figure 4: Ground Control Point (GCP) sample 


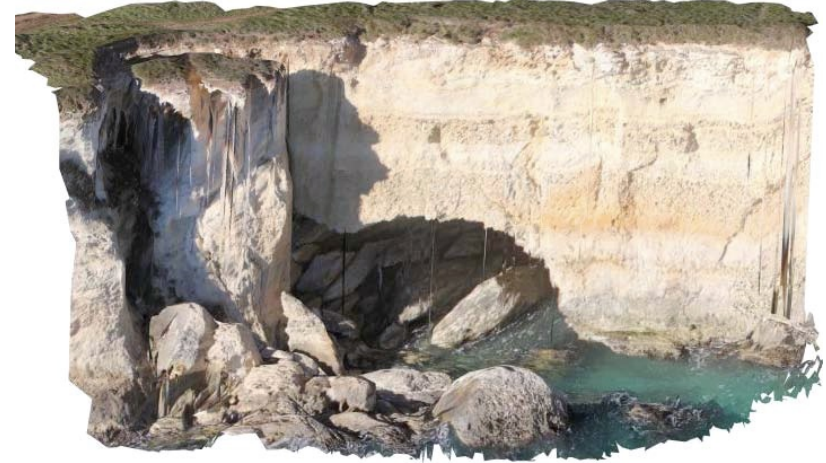

Figure 5: Orthophoto with an arched cavity

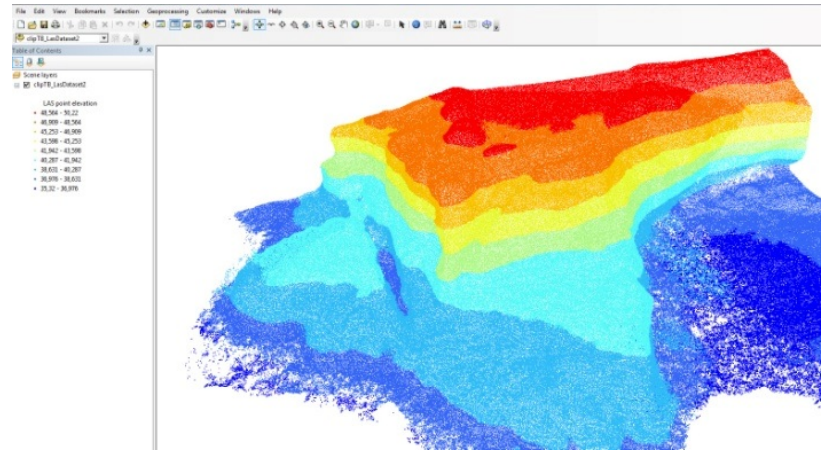

Figure 6: LAS point cloud imported in ArcGis with 9 classes of elevation.

In Figure 5 the orthophoto of a coastal strip of the rocky cliff is shown (zone A in Figure 3). The processed image highlights the presence of a longitudinal fracture that heavily risks the stability of the rocky blocks. In the next step of analysis, the LAS data obtained from AGISOFT PhotoScan have been imported in ArcGis environment. With ArcGis 10.3 a LAS data set has been created. The LAS data have been handled using ArcScene 10.3 and LAS tools (e.g. LAS-clip) provided by RapidolassoGmbH software (Fig. 6). After obtaining the digital model of the coastline (Oliver, 1990), it is possible to extract the features of structural evidence within the geomorphological evolution and then to evaluate instability and rock fall risks. Another interesting component of GIS is its ability to produce pleasing graphics that convey analyses to decision makers and the public at-large. Results are shown in the following. In order to highlight the structural features responsible for the instability of rock masses different layers have been created including different metric characteristics (Figs. 7 and 8). As an example in Figure 7 three layers are

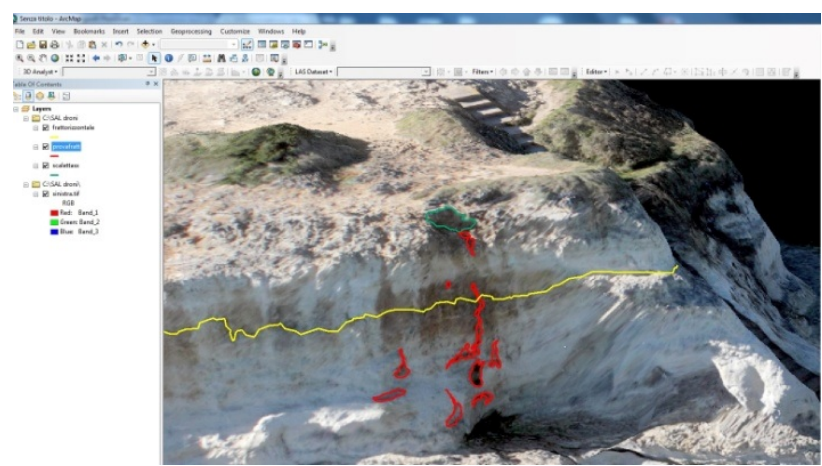

Figure 7: Details of the discontinuity lines

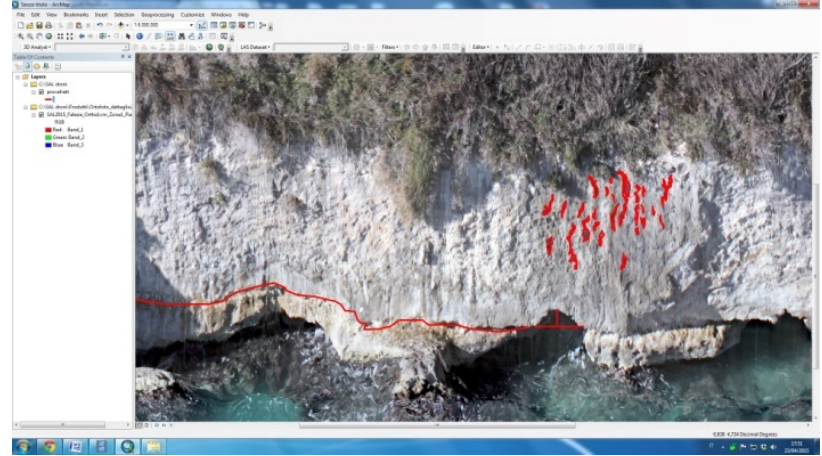

Figure 8: Details of open fractures

marked: open fractures (red lines), surface unevenness (yellow line) and crumbling mass (green line). Analysing these measurements from a geo-structural point of view, the evaluation of the risk of collapse of rock masses can be made and therefore it will be possible to assess the conditions of risk for the safety of people. Furthermore, establishing a reference topographic network based on Ground Control Points that can be assumed stable with respect to the crumbling phenomena, the methodology presented here allows for in time monitoring the evolution of the dangerous elements and then the prediction and prevention of the natural disasters. As a matter of fact, georeferencing the UAV survey, with the same or better accuracy, it is possible to measure the changes of the characteristic parameters and to identify the trend of dangerous phenomena.

\section{THE LIDAR SURVEY}

The cliff were scanned through a LIDAR survey made by the SIT company (Sistemi Informativi Territoriali) on 10 May 2015. The airborne survey were done by using a RIEGL - LMS - Q680 i sensor, with $380 \mathrm{kHz}$ as a frequency, at an altitude of 950 a.s.l. and acquiring about 16.284 .743 points (4-5 points per meter squared for a stripes length of $980 \mathrm{~m}$ ). The final results are shown in Fig. 9.

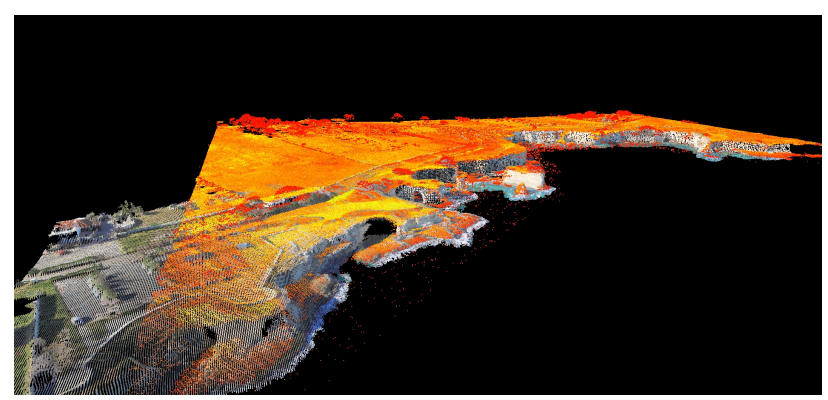

Figure 9: LIDAR survey.

\section{INTRODUCTION TO THE STRUCTURE FROM MOTION TECHNIQUES}

The photogrammetry is a technique used to extract the metrics information of objects starting from the interpretation of photographs. The technique assimilates the photographic images characterized by central projections and defines the position of a point through the intersection of homologous straight lines following the principle of collinearity. In the last few years photogrammetry evolved both in the imaging mode, with the intro- 
duction of digital cameras and new platforms and in the processing techniques. The output that can be obtained today, is a digital 3-D model viewable through point clouds or points in the virtual space, linked to spatial coordinates, and furthermore to RGB values. The cloud points (keypoints) represent the pixels extracted from the alignment of digital images. The Lowe method has been used. It implements a horizontal prioritization process in fully automatic mode by applying multiple algorithms defined as Structure from Motion techniques. Through the algorithm for translational, rotational, scale (SIFT) it is possible to extract (detectors) and to "describe" (descriptors) features of stereoscopic images for the detection of homologous points (keypoint) of larger regions of the image (region detector), overcoming the problems of occlusion and distortion of perspective. For each identified keypoint, it is defined a descriptor (array of numbers) that can describe the radiometric gradients around the point of interest regardless of rotations, scale variations and changes in lighting. Considering the Euclidean distance between these n-dimensional vectors, it is finally possible to identify keypoints homologous between images. The most important feature of the operator SIFT that cannot be found within the other operators, is the robustness to extract and to associate points of interest. The matching operation proposed by Lowe does not ensure robustness in the definition of homologous pairs. So the problem related to the gross errors (outliers) remains, i.e. they need to be removed by appropriate techniques before of the bundle block adjustment procedure. After the identification of the pixels that are equivalent to the homologous points of the shots, the process detects the position of the cameras at the time of the acquisition by the Bundler algorithm considering the point cloud that has been created (called "sparse cloud"). The extraction of the points follows an incremental way. It starts from a couple of shots and then gradually adds other shots to the couple. From this process depends the success of the calculation. Through these already determined correspondences, it is possible to estimate the orientation parameters and to implement the real reconstruction of the object even if camera calibration parameters are unknown. In this way, it is possible to reconstruct the camera positions (Motion) and the geometry of the scene (Structure).

\section{CLOUD TO CLOUD (C2C) METHOD}

The picture below shows how the $\mathrm{C} 2 \mathrm{C}$ method works in its simple version nearest neighbor distance. In this example (Fig. 10), it is assumed that $\mathrm{S} 1$ is the reference surface that has been displaced along the local normal of S1 by a true distance Lt. The Cloud to Cloud method is based on the closest point distance. For small $\mathrm{Lt}, \mathrm{LC} 2 \mathrm{C}$ is dependent on the roughness and point density of PC1 and PC2. An Euclidean distance is calculated for each point of S2. There are other methods of clouds distance computation such
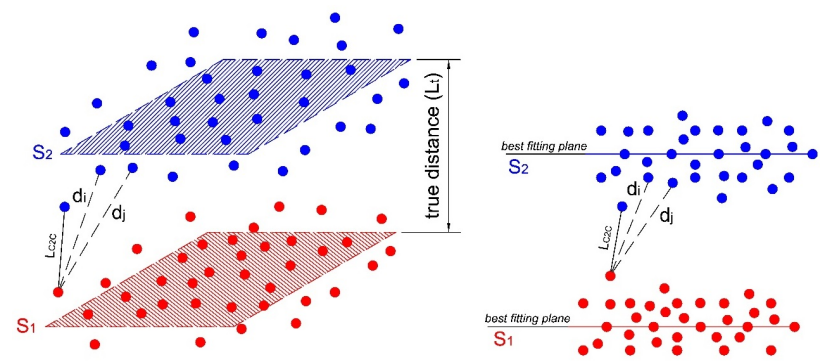

Figure 10: Simplest Cloud-to-Cloud distance.

as Cloud-to-mesh distance (C2M). In this case the method computes distances between a point cloud and a reference 3D mesh or theoretical model.

\section{RESULTS}

The aim of this paper is the comparison between two different point clouds acquired by UAV and LIDAR, respectively. The analysis were done by using the $\mathrm{C} 2 \mathrm{C}$ algorithm which provides good results in terms of Euclidian distances, highlighting differences between the two 3D models. The obtained results are described by the following figures (Fig. 11 and 12).

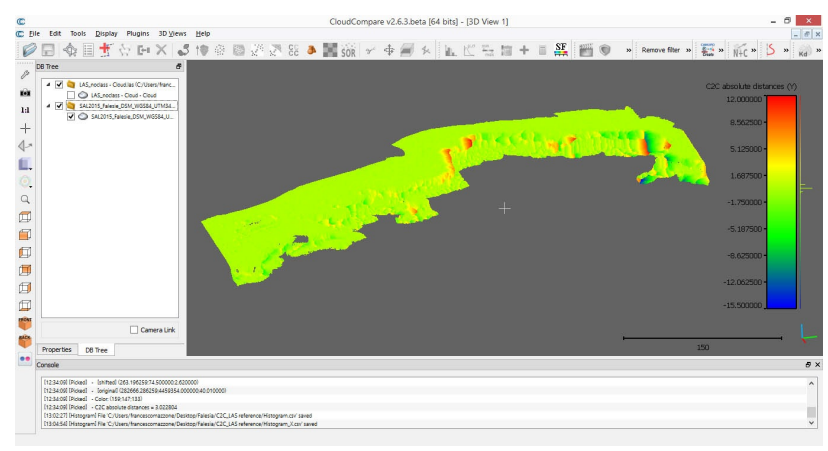

Figure 11: Distances between UAV and LIDAR point clouds.

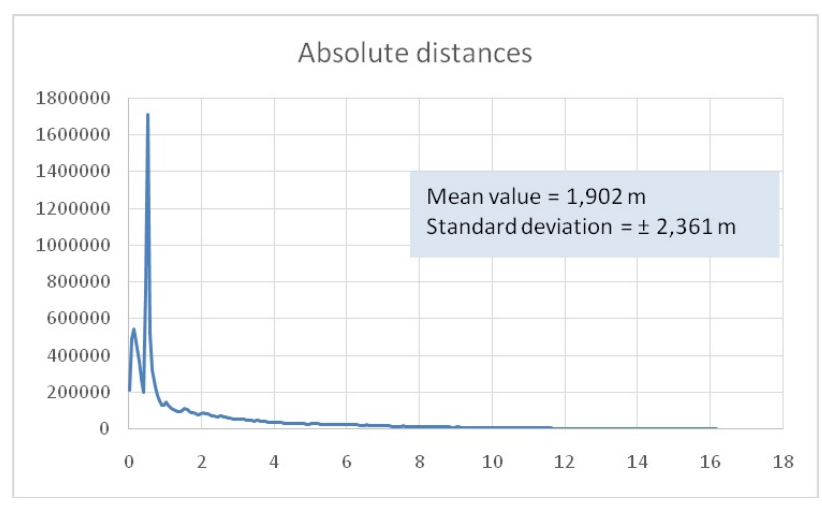

Figure 12: Absolute distances.

\section{CONCLUSION}

UAV photogrammetry opens various new applications in the close range domain, combining aerial and terrestrial photogrammetry, but also introduces low-cost alternatives to the classical manned aerial photogrammtery. The platform is equipped with a photogrammetric measurement system. Current standard UAVs allow the registration and tracking of the position and orientation of the implemented sensors in a local or global coordinate system. Hence, UAV photogrammetry can be understood as a new photogrammetric measurement tool.

\section{ACKNOWLEDGEMENTS}

This work was done thanks to the SAL engineering and SIT company support.

\section{REFERENCES}

Burrough,,P.A., 1986. Principles of Geographical Information Systems for Land Resources Assessment. Oxford University, New York. 
Caprioli, M., Mancini, F., Mazzone, F., Scarano, M., Trizzino, R., 2015. UAV Surveys for Representing and Document the Cultural Heritage. XIII International Forum Le Vie dei Mercanti Heritage and Technology. Aversa-Capri, Italy, June 11-13, 477-484.

Hoek, E., and Bray,J., 1977. Rock Slope Engineering, 1st Edition IMM, London.

Oliver, M. A., 1990. Kriging: A Method of Interpolation for Geographical Information Systems. International Journal of Geographic Information Systems, 4: 313332.

Terzopoulos,D., 1988. The computation of visible-surface representations. IEEE Transactions on Pattern Analysis and Machine Intelligence, 10: 417438.

Watson D. F., Philip, G. M., 1985. A Refinement of Inverse Distance Weighted Interpolation. Geoprocessing, 2:315327.

Zhang, C., 2009. Photogrammetric processing of low-altitude UAV imagery. ASPRS 2009 Annual Conference, Baltimore, Maryland (U.S.). 